\title{
Influencia de la alteración meteórica de dos carbones coquizables con contenido medio de materia volátil en la calidad del coque producido $^{(\bullet)}$
}

\author{
M.D. Casal $^{(*)}$, M.A. Díez ${ }^{(*)}$, R. Álvarez ${ }^{(*)}$, G. Barriocanal $^{(*)}$, C.S. Canga ${ }^{(*)}$ y J.J. Pis ${ }^{(*)}$
}

Resumen Se presenta el estudio de la alteración meteórica de dos carbones bituminosos con contenido medio de materia volátil y de diferente origen geográfico, que han sido almacenados a la intemperie en el Parque del INCAR. Ambos carbones son constituyentes de las mezclas de carbones coquizables utilizadas por la Corporación Siderúrgica Integral (CSI) para la producción de coque de horno alto. Entre las técnicas utilizadas para detectar el grado de meteorización de dichos carbones, la fluidez máxima Gieseler es el indicador más sensible para detectar lá pérdida de propiedades plásticas. Ambas series de carbones presentan diferente comportamiento frente a la meteorización. Si bien la alteración meteórica provoca una pérdida de fluidez máxima con el tiempo de almacenamiento, debido a una pérdida de hidrógeno alifático en los dos carbones, ésta se produce a diferente velocidad. Estas diferencias se ponen de manifiesto en la calidad de los coques producidos en la planta de coquización del INCAR (escala semiindustrial). La serie de coques producidos a partir del carbón inicialmente menos fluido y que experimenta una pérdida a mayor velocidad empeora claramente, mientras que los coques producidos a partir del carbón más fluido presentan una ligera mejora inicial, para terminar empeorando. Estos resultados ponen en evidencia que la alteración meteórica no sólo afecta positivamente en el caso de algunos carbones con contenido elevado de materia volátil, como ya se había detectado en estudios realizados por otros autores, sino también a determinados carbones con contenido medio de materia volátil.

Palabras clave: Carbón. Meteorización. Coquización. Calidad del coque.

\section{Influence of natural weathering of two medium-volatile bituminous coals of similar rank on coke quality}

\begin{abstract}
The study of the weathering of two bituminous coals stored at the INCAR stockyard and its influence on coke quality is presented. These coals of similar rank are present in industrial blends used by the Corporación Siderúrgica Integral (CSI) for the production of blast-furnace coke. Of all the techniques used to determine the degree of weathering, via thermoplastic properties, Gieseler plastometry was found to be the most sensitive. Coking tests were carried out in the semi-industrial coking plant at INCAR. The effect of weathering on each coal series is completely different. Weathering produces a loss of maximum fluidity accompanied by a decrease in aliphatic hydrogen in both coals. However, the loss rate is different in each case. Coke quality clearly decreases in one, while in the other improves at first but then gradually deteriorates. An improvement in coke quality due to weathering not only affects some high-volatile coals as it is well known but also mediumvolatile coals.
\end{abstract}

Keywords: Coal. Weathering. Coking. Coke quality.

\section{INTRODUCCIÓN}

(•) Trabajo recibido el día 18 de abril de 1997.

(*) Instituto Nacional del Carbón (INCAR), (CSIC). Aptdo. 73. 33080-Oviedo (España).
Las condiciones atmosféricas pueden alterar las propiedades del carbón y, consecuentemente, 
modificar su comportamiento durante el proceso de coquización afectando a la calidad del coque obtenido. Este hecho hace que exista gran interés en el estudio de los cambios químico-estructurales de la materia orgánica del carbón y de la variación de las propiedades coquizantes, como consecuencia de la interacción del carbón con los agentes atmosféricos ( 1 y 2 ).

La meteorización se produce, principalmente, como resultado de la inestabilidad termodinámica que existe entre el carbón y los componentes del ambiente que lo rodean. Se trata de un fenómeno complejo que implica varios procesos químicos simultáneos, acompañados la mayoría de las veces por cambios físicos (3). Durante este proceso tiene lugar una oxidación debida al aire, a la humedad y a la radiación UV, que se puede producir de manera uniforme o local a una velocidad continua o discontinua. Así, se comprende que resulte difícil reproducir artificialmente en laboratorio los procesos que tienen lugar de manera natural como consecuencia de la interacción del carbón con el ambiente.

La alteración del carbón se puede producir en el propio yacimiento, durante su almacenamiento y transporte entre la mina y el lugar de utilización y en minas abandonadas y escombreras. La necesidad de almacenamiento, antes y/o después de su transporte, conlleva interacciones con los agentes atmosféricos que producen modificaciones en la matriz orgánica del carbón. Como consecuencia de ello, se alteran sus propiedades termoplásticas y su comportamiento durante el proceso de coquización (46). El carbón, cuando se somete a tratamiento térmico en atmósfera inerte, pasa por diferentes etapas en su transición a coque, siendo, sin lugar a dudas, la etapa entre $350-500{ }^{\circ} \mathrm{C}$ la etapa clave de la formación de la estructura cristalina y porosa del coque y, consecuentemente, la que define las propiedades del coque, tales como resistencia mecánica en frío y reactividad frente a $\mathrm{CO}_{2}$. En esta etapa, el carbón reblandece/funde pasando por un estado plástico donde las partículas individuales interaccionan para formar una masa fluida. Es en este momento cuando el carbón desarrolla la máxima fluidez en el proceso, existiendo una movilidad de las moléculas que facilita las reacciones intermoleculares de condensación aromática y el ordenamiento de las mismas. A partir de este momento, la fluidez del sistema comienza a descender y, a una temperatura próxima a $500{ }^{\circ} \mathrm{C}$, la masa de reacción resolidifica dando lugar a un residuo sólido denominado semicoque o coque verde. Los cambios físicos que tienen lugar en la denominada etapa plástica se ponen en evidencia mediante ensayos realizados en plastómetros y dilatómetros. Así, pues, se pueden esperar distintos comportamientos dependiendo de las características intrínsecas del carbón y de las condiciones medioambientales, lo cual se verá reflejado en una mejora o empeoramiento de la calidad del coque obtenido.

Este fenómeno de alteración meteórica viene siendo estudiado desde hace tiempo, pero la mayoría de los trabajos realizados se basan en oxidaciones artificiales (7-11) o utilizan condiciones climáticas extremas que no se asemejan al clima suave de Asturias, en cuyo contexto se sitúa la presente investigación. Es preciso señalar el interés que tiene esta investigación para la industria siderúrgica, en la que se coquizan mezclas que se preparan cada cierto tiempo, en función de los distintos carbones de que se dispone en ese momento.

En trabajos anteriores se realizó un estudio de la influencia de la alteración meteórica sobre una mezcla industrial constituida por trece carbones de diferente rango, llegándose a la conclusión de que podía estar almacenada durante un año sin efectos negativos importantes sobre la calidad del coque (12). Las pequeñas variaciones que experimentan ciertas propiedades de una mezcla industrial durante períodos relativamente cortos de almacenamiento $\mathrm{y}$, en consecuencia, los parámetros de calidad de los coques resultantes se deberán a la alteración de los carbones individuales que la constituyen y/o al resultado de efectos contrarios y compensatorios de la exposición a la intemperie de dichos constituyentes. Por consiguiente, se requiere un estudio de los carbones individuales que permita explicar tales fenómenos y, por otra parte, optimizar el período máximo de almacenamiento de un determinado carbón en el parque.

A continuación, se presenta el estudio correspondiente a dos de los trece carbones que componen la mezcla industrial anteriormente estudiada (12). Ambos carbones tienen similar contenido de materia volátil y distinta procedencia geográfica. El objetivo de este trabajo ha sido establecer la influencia de la alteración meteórica de dichos carbones almacenados en condiciones climáticas suaves, en sus propiedades coquizantes, en el proceso de coquización y en la calidad del coque obtenido. Esto permitirá optimizar el período de almacenamiento de los mismos, antes de que la calidad del coque comience a deteriorarse, y explicar además, al menos parcialmente, el comportamiento de la mezcla industrial frente a la alteración meteórica.

\section{PARTE EXPERIMENTAL}

En el Parque de Carbones del INCAR se almacenaron dos carbones bituminosos con similar contenido de materia volátil y diferente origen (Canadiense -Ca- y Australiano - $\mathrm{Au}-$ ) en pilas de $50 \mathrm{t}$ durante un período de 5-6 meses. A intervalos apropiados de tiempo se tomaron muestras para análisis y ensayos de carbonización. Se siguió el grado de 
alteración de cada carbón utilizando parámetros deducidos del análisis inmediato (contenido de materia volátil), IRTF, índice de hinchamiento en crisol, IHC, (ISO 501-1981), ensayo dilatométrico Arnu (ISO 349) y ensayo de fluidez Gieseler (ASTM D2639).

En el ensayo dilatométrico Arnu, el carbón se somete a calentamiento en ausencia de aire y se obtiene una medida de la variación de volumen que se produce. El parámetro $b$, obtenido en este ensayo, proporciona una medida de la dilatación máxima. El valor de la dilatación Arnu se puede expresar como $\alpha(\alpha= \pm(34+b)(13)$. En este trabajo, y con objeto de poder reflejar las variaciones de dilatación Arnu en porcentaje de pérdida con respecto al valor inicial, se ha utilizado el valor $\alpha+34$ al que se ha denominado hinchamiento absoluto Arnu.

El ensayo de fluidez Gieseler proporciona una medida de la variación de la fluidez del carbón cuando se somete a calentamiento $(3 \mathrm{~K} / \mathrm{min})$. De este ensayo se obtienen los siguientes parámetros:

a) Temperatura de reblandecimiento $\left(T_{\mathrm{r}}\right)$, que es aquella a la que el carbón empieza a ser fluido;

b) Temperatura de fluidez máxima $\left(T_{\mathrm{f}}\right)$, definida como la temperatura a la cual el carbón alcanza el máximo valor de fluidez;

c) Temperatura de resolidificación $\left(T_{\mathrm{s}}\right)$, que es aquella a la que se produce la resolidificación del residuo y coincide con el cese de movimiento del dial;

d) Intervalo plástico, que se define como la diferencia entre la temperatura de resolidificación y reblandecimiento;

e) Fluidez máxima $\left(F_{\mathrm{m}}\right)$ que se mide en divisiones de dial por minuto (ddpm).

La evaluación semicuantitativa de hidrógeno aromático y alifático en la matriz orgánica de los carbones se realizó mediante espectroscopía infrarroja por transformada de Fourier (IRTF), utilizando un espectrómetro Perkin-Elmer modelo 1750. Se utilizó la fracción natural $<0,15 \mathrm{~mm}$ obtenida al tamizar la muestra general de cada carbón sin molienda y realizando la toma de muestra de acuerdo con la norma internacional ISO 1988-1975. Las muestras se prepararon en pastillas convencionales utilizando bromuro de potasio $(\mathrm{KBr})$ como matriz, en proporción $1 \mathrm{mg}$ carbón/100 mg KBr. Como paso previo, las pastillas de carbón-KBr se secaron en una estufa a $120{ }^{\circ} \mathrm{C}$ hasta peso constante. Los espectros se normalizaron al equivalente a $1 \mathrm{mg}$ de muestra en base seca y libre de cenizas, utilizando un factor de normalización definido como el cociente del área de la pastilla, constante para todas las muestras, y el peso de la muestra de carbón libre de humedad y cenizas, expresado en mg (14 y 15). El estudio semicuantitativo de la distribución de hidrógeno aromático y alifático se realizó mediante la medida de la absorbancia máxima de las bandas centradas a 3.040 y $2.920 \mathrm{~cm}^{-1}$, respectivamente.

Los ensayos de coquización, tanto de las muestras de carbón fresco como alterado, se llevaron a cabo en un horno de $6 \mathrm{t}$ de capacidad $(450 \mathrm{~mm}$ de anchura, $6,9 \mathrm{~m}$ de longitud y $3 \mathrm{~m}$ de altura), ubicado en la Planta Experimental de Coquización del INCAR, que opera a escala semiindustrial. La temperatura media de calentamiento para todos los ensayos de coquización fue $1.240 \pm 15^{\circ} \mathrm{C}$ y el tiempo de coquización estuvo comprendido entre 18 y $19 \mathrm{~h}$. Se intentaron reproducir, en la medida de lo posible, las condiciones de coquización utilizadas por una batería industrial de hornos de coque.

La calidad del coque se determinó mediante el ensayo de resistencia mecánica (IRSID) (ISO 5561980), definiéndose dos índices: el de cohesión, $I_{20}$, que corresponde al porcentaje en peso de coque de tamaño mayor de $20 \mathrm{~mm}$, y el índice de abrasión, $I_{10}$, definido como el porcentaje en peso de coque de tamaño menor de $10 \mathrm{~mm}$.

Para llevar a cabo la evaluación de la reactividad del coque frente a $\mathrm{CO}_{2}(\mathrm{CRI})$ y la resistencia mecánica del residuo después de reacción (CSR), se utilizó el método de Nippon Steel Coorporation (NSC) (16). La reactividad del coque frente a $\mathrm{CO}_{2}(\mathrm{CRI})$ se determinó a $1.100{ }^{\circ} \mathrm{C}$ durante $2 \mathrm{~h}$ con un flujo de $\mathrm{CO}_{2}$ de $5 \mathrm{~L} / \mathrm{min}$. La muestra de coque procedente de este ensayo de reactividad se somete al de resistencia mecánica, que consiste en dar 600 vueltas en un tambor a una velocidad de $20 \mathrm{rpm}$. El índice de reactividad (CRI) se expresa como el porcentaje de pérdida de peso del coque después de reacción, y la resistencia mecánica después de reacción (CSR) como el porcentaje en peso de coque que queda mayor de $10 \mathrm{~mm}$ después de someter la muestra de coque procedente del ensayo de reactividad a un tratamiento de 600 vueltas en un tambor a una velocidad de $20 \mathrm{rpm}$.

\section{ANÁLISIS DE LOS RESULTADOS}

\subsection{Efecto de la alteración meteórica sobre la estructura del carbón y sus propiedades coquizantes}

En la tabla I se muestran los resultados del análisis petrográfico de los dos carbones iniciales de diferente origen geográfico $(\mathrm{Ca}$ y $\mathrm{Au})$. Se observa que la composición maceral es muy similar, al igual que los parámetros de rango tales como la reflectancia media de la vitrinita, $R_{\mathrm{o}}$, (Tabla I) y el contenido de materia volátil (Tabla II).

Las principales características de los carbones originales $(\mathrm{Ca}, \mathrm{Au})$ y de los carbones a diferente tiempo de almacenamiento se muestran en la tabla 
TABLA I.- Análisis petrográfico de los dos carbones iniciales

TABLE I.- Petrographic analysis of the coals as received

\begin{tabular}{|l|c|c|}
\hline \multicolumn{1}{|c|}{ Carbón } & Ca & $\mathrm{Au}$ \\
\hline Origen del carbón & Canadá & Australia \\
$\bar{R}_{\mathrm{o}}, \%$ & 1,14 & 1,14 \\
Desviación estándar de $\bar{R}_{\mathrm{o}}$ & 0,12 & 0,15 \\
\hline \multicolumn{3}{|c|}{ Contenido en macerales, } \\
\% vol.* \\
\hline Vitrinita & 74,2 & 77,3 \\
Exinita & 0,00 & 0,00 \\
Semifusinita & 19,2 & 15,1 \\
Fusinita & 6,6 & 7,6 \\
\hline
\end{tabular}

* Expresado libre de materia mineral.

II. Inicialmente, la fluidez máxima Gieseler $\left(F_{\mathrm{m}}\right)$ para los dos carbones es muy diferente. Así, el carbón $\mathrm{Ca}$ es bituminoso y poco fluido (30 ddpm), mientras que el carbón Au presenta mayor fluidez (639 ddpm). En cuanto al intervalo plástico, en principio es similar para ambos carbones, si bien hay que destacar que las temperaturas de reblandecimiento $\left(T_{\mathrm{r}}\right)$ y solidificación $\left(T_{\mathrm{s}}\right)$ para el carbón $\mathrm{Au}$ son ligeramente más bajas que para el carbón $\mathrm{Ca}$ (Tabla II). Para las dos series de carbones, la fluidez máxima Gieseler $\left(F_{\mathrm{m}}\right)$ disminuye con el tiempo de almacenamiento, pero lo hace a diferentes velocidades. Con objeto de evaluar la pérdida relativa de fluidez con el tiempo de almacenamiento, se ha utilizado como parámetro de medida el porcentaje de pérdida de fluidez máxima con respecto al valor inicial. En la figura 1 se representa dicho parámetro en función del tiempo de almacenamiento. Se observa que dentro de cada serie y para el período de tiempo estudiado, la velocidad de pérdida de fluidez no es uniforme, sino que se produce en varias etapas. Después de un período de almacenamiento de 49 días, la serie correspondiente al carbón $\mathrm{Ca}$, poco fluido inicialmente, pierde un $60 \%$ de la $F_{\mathrm{m}}$ inicial, mientras que la serie correspondienté al carbón $\mathrm{Au}$, de mayor fluidez, sólo pierde un $11 \%$ y, aproximadamente, el $20 \%$ a los 76 días.

TABLA II.- Principales características de los carbones originales y alterados meteóricamente

TABLE II.-Main characteristics of the coals, as received and weathered

\begin{tabular}{|l|c|c|c|c|c|}
\hline \multicolumn{6}{|c|}{ Carbón canadiense -Ca- } \\
\hline Tiempo de almacenamiento, & 0 & 49 & 91 & 138 & 180 \\
días & 0,4 & 9,4 & 9,4 & 9,5 & 9,6 \\
\hline Cenizas, \% bs & 0,43 & 0,39 & 0,41 & 0,48 & 0,43 \\
Azufre, \% bs & 22,1 & 22,1 & 22,0 & 21,8 & 21,9 \\
Materia volátil, \% bs & 7 & $71 / 4$ & $63 / 4$ & $41 / 2$ & 5 \\
IHC & 30 & 12 & 9 & 10 & 5 \\
$F_{\mathrm{m}}$ Gieseler, ddpm & 414 & 429 & 430 & 433 & 433 \\
$T_{\mathrm{r}},{ }^{\circ} \mathrm{C}$ & 498 & 489 & 490 & 486 & 484 \\
$T_{\mathrm{s}}{ }^{\circ} \mathrm{C}$ & 84 & 60 & 60 & 53 & 51 \\
Intervalo plástico, ${ }^{\circ} \mathrm{C}$ & $\mathrm{Carbón}$ australiano-Au- & & & \\
\hline \multicolumn{7}{|c|}{} & & & & \\
\hline Tiempo de almacenamiento, & 0 & 41 & 76 & 119 & 156 \\
días & 10,4 & 11,1 & 9,8 & 9,8 & 10,0 \\
\hline Humedad, \% & 9,9 & 10,0 & 9,6 & 9,4 & 9,9 \\
Cenizas, \% bs & 0,60 & 0,58 & 0,60 & 0,61 & 0,59 \\
Azufre, \% bs & 23,2 & 23,5 & 23,5 & 23,4 & 23,3 \\
Material volátil, \% bs $_{\text {IHC }}$ & $71 / 2$ & $71 / 2$ & $71 / 2$ & $71 / 4$ & 7 \\
$F_{\mathrm{m}}$ Gieseler, ddpm & 639 & 568 & 510 & 252 & 287 \\
$T_{\mathrm{r}},{ }^{\circ} \mathrm{C}$ & 406 & 408 & 408 & 409 & 409 \\
$T_{\mathrm{s}},{ }^{\circ} \mathrm{C}$ & 489 & 491 & 491 & 491 & 492 \\
Intervalo plástico, ${ }^{\circ} \mathrm{C}$ & 83 & 83 & 83 & 82 & 83 \\
\hline
\end{tabular}




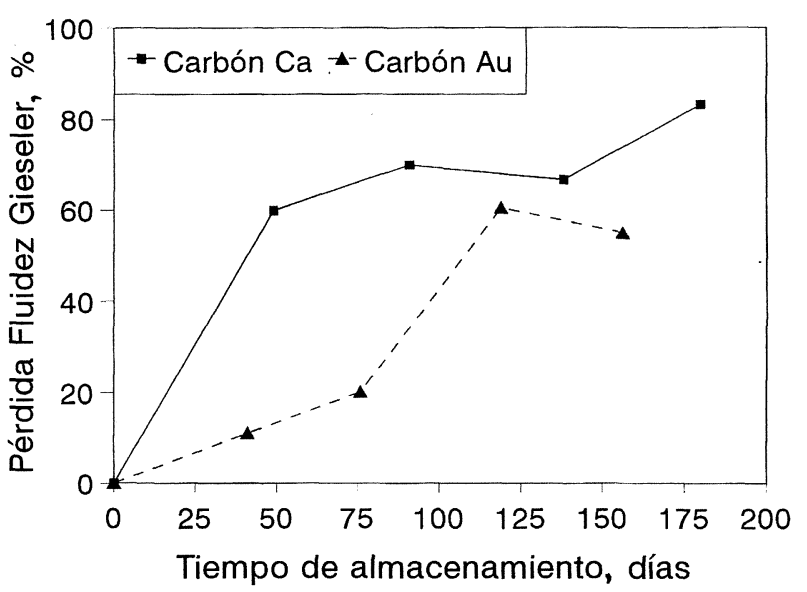

FIG. 1.- Variación de la pérdida de $F_{\mathrm{m}}$ Gieseler con el tiempo de almacenamiento.

FIG. 1.- Variation of the loss of Gieseler $\mathrm{F}_{\mathrm{m}}$ with weathering time.

En el estado intermedio de almacenamiento, la $F_{\mathrm{m}}$ de la serie $\mathrm{Ca}$ disminuye lentamente, mientras que la serie Au muestra una pérdida más pronunciada de la $F_{\mathrm{m}}$ después de 76 días de exposición a la intemperie. En el último estado de almacenamiento (>140 días), la pérdida de $F_{\mathrm{m}}$ para la serie $\mathrm{Ca}$ representa el $83 \%$ del valor inicial, mientras que la serie Au después de 156 días pierde un $60 \%$. Por tanto, el carbón $\mathrm{Au}$, que es el que posee una $F_{\mathrm{m}}$ inicial mayor, es el que presenta menor pérdida de plasticidad con el tiempo de almacenamiento, si bien es una pérdida bastante significativa.

El intervalo plástico para las dos series de carbones estudiadas presenta también comportamientos diferentes. Mientras que la serie Ca muestra una disminución del intervalo plástico, debida a un aumento de la temperatura de reblandecimiento $\left(T_{\mathrm{r}}\right)$ y a una disminución de la temperatura de resolidificación $\left(T_{\mathrm{s}}\right)$, en la serie Au no se modifica dicho intervalo, ya que tanto la $T_{\mathrm{r}}$ como la $T_{\mathrm{s}}$ no varían prácticamente con el tiempo de almacenamiento (Tabla II).

Las propiedades plásticas de los carbones también se miden utilizando ensayos dilatométricos que proporcionan información acerca del hinchamiento que experimenta el carbón cuando se somete a calentamiento en ausencia de aire. La figura 2 muestra la variación de la pérdida de hinchamiento absoluto Arnu con el tiempo de almacenamiento. El comportamiento de las dos series de carbones es diferente, tal y como ocurría en el ensayo Gieseler, siendo el carbón Ca más susceptible a la pérdida de capacidad de hinchamiento que el carbón Au. Así, el carbón Ca pierde un $20 \%$ de hinchamiento para un tiempo de almacenamiento de 49 días, mientras que el carbón Au experimenta una pequeña pérdida hasta los 76 días $(10 \%)$. A partir de estos períodos, la serie del carbón Ca sigue perdiendo capacidad de

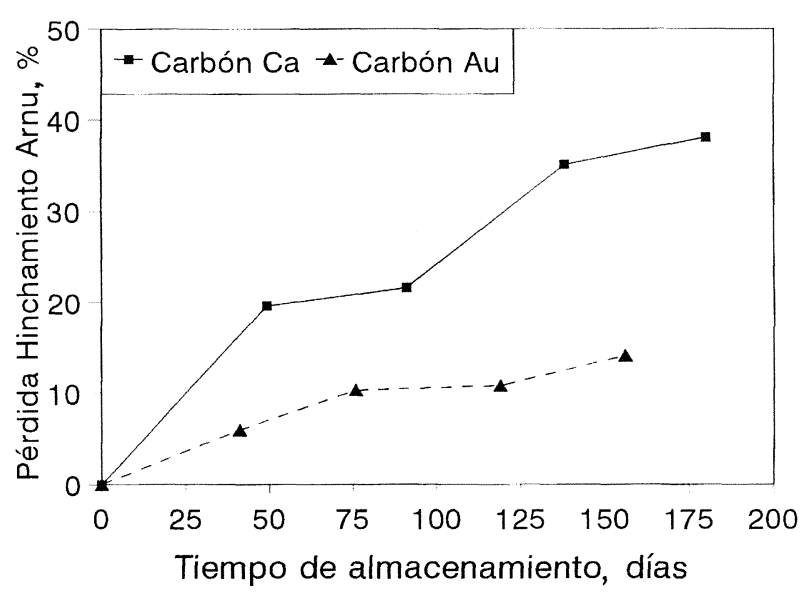

FIG. 2.- Variación de la pérdida de hinchamiento Arnu con el tiempo de almacenamiento.

\section{FIG. 2.-Variation of the loss of Arnu dilatation with weathering time.}

hinchamiento de forma más suave, mientras que la serie Au tiende a estabilizarse desde los 76 días hasta el final del período de almacenamiento.

A pesar de que el ensayo de hinchamiento en crisol (IHC) es menos sensible al fenómeno de alteración meteórica, se observa una disminución de dicho índice, siendo más significativa en las últimas etapas de almacenamiento del carbón $\mathrm{Ca}$. Por otro lado el IHC, para la serie $\mathrm{Au}$, permanece prácticamente constante (Tabla II).

El estudio realizado por IRTF muestra variaciones de la intensidad relativa de las bandas a 3.040 $\mathrm{cm}^{-1}$, correspondiente a la vibración de tensión del enlace $\mathrm{C}-\mathrm{H}$ aromático, y a $2.920 \mathrm{~cm}^{-1}$, correspondiente a la vibración de tensión del enlace $\mathrm{C}$ - $\mathrm{H}$ alifático. Estas diferencias se ponen de manifiesto en la relación H3040/H2920 de los carbones de cada serie. La tendencia es similar, mostrando una disminución de hidrógeno alifático (aumento de la relación H3040/H2920) con el tiempo de almacenamiento, hasta el período correspondiente a 91 días para la serie Ca y a 119 días para la serie Au (Fig. 3). Sin embargo, existen diferencias sustanciales en la evolución de los dos carbones, tal y como ocurre en la pérdida de $F_{\mathrm{m}}$ Gieseler, sugiriendo diferente reactividad de los grupos alifáticos frente al oxígeno. La disminución de los grupos alifáticos es siempre más lenta para la serie Au. Así, se puede explicar parcialmente la menor velocidad de pérdida de fluidez como consecuencia de la diferente reactividad de los grupos alifáticos.

\subsection{Efectos de la alteración meteórica sobre las propiedades del coque}

En la tabla III se muestran las condiciones de coquización utilizadas para los distintos ensayos de 


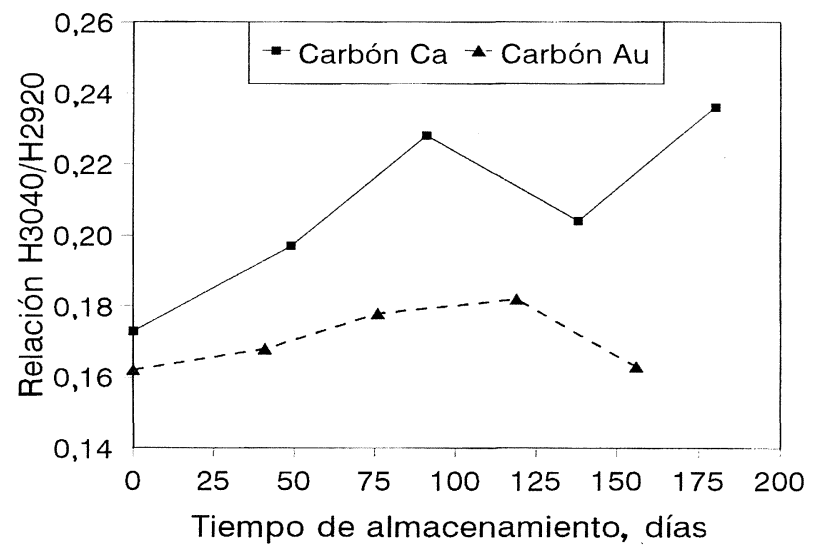

FIG. 3.- Variación de la relación H3040/H2920 con el tiempo de almacenamiento.

FIG. 3.- Variation of the ratio H3040/H2920 with weathering time.

carbonización. Como ya se comentó anteriormente, en todos los casos se intentó reproducir en la medida de lo posible las condiciones industriales.

En la tabla IV se observan las principales características de los coques metalúrgicos producidos en el horno de $6 \mathrm{t}$, a partir de las dos series de carbones estudiadas.
Con respecto a la granulometría del coque, la alteración meteórica produce dos efectos contrarios. Tanto el tamaño medio como el tamaño útil (20-80 $\mathrm{mm}$ ) no presentan una tendencia clara para la serie de coques procedentes del carbón Ca. Sin embargo, sí parece existir un ligero aumento de la producción de finos de coque $(<20 \mathrm{~mm})$ con el tiempo de almacenamiento. En la serie Au se observa, en general, un aumento del tamaño medio acompañado por un aumento de la fracción de tamaño $>80 \mathrm{~mm}$ y una disminución de la fracción del tamaño útil (20-80 $\mathrm{mm})$ a medida que aumenta el tiempo de almacenamiento (Tabla IV).

Los coques procedentes de la serie Ca muestran una disminución progresiva del índice de cohesión IRSID $\left(I_{20}\right)$ junto con el correspondiente aumento del índice de abrasión IRSID $\left(I_{10}\right)$, a medida que aumenta el tiempo de exposición a la intemperie (Fig. 4). Se debe comentar que los coques procedentes de la serie Au muestran una ligera mejoría, tanto del $I_{20}$ como del $I_{10}$ hasta el período correspondiente a los 76 días de almacenamiento, deteriorándose después de este punto, pero llegando a alcanzar valores similares a los de la muestra de coque original (Fig. 4). De estos resultados se deduce que en las primeras etapas de almacenamiento se

TABLA III.- Condiciones de coquización en la producción de los coques estudiados

TABLE III.-Coking conditions used in the manufacture of the cokes studied

\begin{tabular}{|c|c|c|c|c|c|}
\hline \multicolumn{6}{|c|}{ Carbón canadiense $-\mathrm{Ca}-$} \\
\hline Tiempo de almacenamiento, días & 0 & 49 & 91 & 138 & 180 \\
\hline Granulometría del carbón, \% & & & & & \\
\hline$>3 \mathrm{~mm}$ & 6,5 & 5,4 & 4,8 & 6,0 & 6,0 \\
\hline$<2 \mathrm{~mm}$ & 80,8 & 80,4 & 82,9 & 79,0 & 81,1 \\
\hline$<0,5 \mathrm{~mm}$ & 45,7 & 44,8 & 47,9 & 43,9 & 46,3 \\
\hline Condiciones de coquización & & & & & \\
\hline Densidad carga, $\mathrm{kg} / \mathrm{m}^{3} \mathrm{bs}$ & 692 & 700 & 675 & 687 & 662 \\
\hline Temper. media calentamiento, ${ }^{\circ} \mathrm{C}$ & 1.253 & 1.255 & 1.246 & 1.229 & 1.251 \\
\hline Tiempo coquización, (h:min) & $18: 30$ & $18: 30$ & 19:10 & $18: 10$ & 18:00 \\
\hline \multicolumn{6}{|c|}{ Carbón australiano - Au- } \\
\hline Tiempo de almacenamiento, días & 0 & 41 & 76 & 119 & 156 \\
\hline Granulometría del carbón, \% & & & & & \\
\hline$>3 \mathrm{~mm}$ & 12,9 & 13,7 & 13,3 & 13,6 & 13,9 \\
\hline$<2 \mathrm{~mm}$ & 74,8 & 74,7 & 74,4 & 74,7 & 74,1 \\
\hline$<0,5 \mathrm{~mm}$ & 31,1 & 31,7 & 33,1 & 31,6 & 33,0 \\
\hline Condiciones de coquización & & & & & \\
\hline Densidad carga, $\mathrm{kg} / \mathrm{m}^{3} \mathrm{bs}$ & 697 & 711 & 698 & 698 & 699 \\
\hline Temper. media calentamiento, ${ }^{\circ} \mathrm{C}$ & 1.251 & 1.238 & 1.235 & 1.230 & 1.245 \\
\hline Tiempo coquización, (h:min) & 18:00 & $18: 30$ & 18:00 & $18: 34$ & 18:00 \\
\hline
\end{tabular}


TABLA IV.- Principales características de los coques obtenidos a partir de los carbones alterados

TABLE IV.-Main characteristics of the cokes obtained from the weathered coals

\begin{tabular}{|c|c|c|c|c|c|}
\hline \multicolumn{6}{|c|}{ Carbón canadiense $-\mathrm{Ca}-$} \\
\hline $\begin{array}{l}\text { Tiempo de almacenamiento } \\
\text { del carbón, días }\end{array}$ & 0 & 49 & 91 & 138 & 180 \\
\hline Cenizas, $\%$ bs & 11,8 & 11,8 & 11,5 & 11,8 & 11,7 \\
\hline Volátiles, \% bs & 0,1 & 0,1 & 0,1 & 0,3 & 0,2 \\
\hline Azufre, $\%$ bs & 0,35 & 0,33 & 0,35 & 0,31 & 0,32 \\
\hline Granulometría, \% & & & & & \\
\hline$>80 \mathrm{~mm}$ & 39,9 & 43,1 & 46,5 & 40,4 & 38,2 \\
\hline $20 / 80 \mathrm{~mm}$ & 55,8 & 50,5 & 46,8 & 52,5 & 53,5 \\
\hline$<20 \mathrm{~mm}$ & 4,3 & 6,4 & 6,7 & 7,0 & 8,3 \\
\hline Tamaño medio, mm & 74,9 & 81,6 & 77,4 & 73,9 & 71,2 \\
\hline \multicolumn{6}{|c|}{ Carbón australiano -Au- } \\
\hline $\begin{array}{l}\text { Tiempo de almacenamiento } \\
\text { del carbón, días }\end{array}$ & 0 & 41 & 76 & 119 & 156 \\
\hline Cenizas, $\%$ bs & 12,6 & 12,5 & 12,1 & 12,0 & 12,5 \\
\hline Volátiles, \% bs & 0,4 & 0,4 & 0,2 & 0,7 & 0,3 \\
\hline Azufre, $\%$ bs & 0,54 & 0,53 & 0,54 & 0,55 & 0,52 \\
\hline Granulometría, \% & & & & & \\
\hline$>80 \mathrm{~mm}$ & 54,8 & 57,8 & 57,9 & 61,6 & 60,6 \\
\hline $20 / 80 \mathrm{~mm}$ & 40,9 & 38,1 & 38,3 & 33,3 & 35,1 \\
\hline$<20 \mathrm{~mm}$ & 4,3 & 4,1 & 3,9 & 5,1 & 4,4 \\
\hline Tamaño medio, mm & 84,4 & 85,5 & 86,6 & 89,3 & 97,2 \\
\hline
\end{tabular}

produce una mejoría de la resistencia mecánica del coque para la serie Au.

$\mathrm{Al}$ igual que para los índices IRSID, el efecto de la alteración meteórica sobre la reactividad frente a $\mathrm{CO}_{2}$ (índice CRI) y la resistencia mecánica después de dicha reacción (índice CSR), es bastante diferente para ambas series de carbones estudiadas. Así, el CRI aumenta con el tiempo de almacenamiento para los coques procedentes de la serie $\mathrm{Ca}$, mientras que para los coques de la serie $\mathrm{Au}$, en principio, se produce una ligera disminución del índice CRI hasta el período correspondiente a los 76 días de almacenamiento (Fig. 5). A partir de este momento, se llegan a alcanzar valores de CRI más elevados que el correspondiente al coque inicial de dicha serie. Este comportamiento se confirma con los valores del índice CSR, donde se observa un claro deterioro de los coques procedentes de la serie $\mathrm{Ca}$ (disminución del índice CSR) y una ligera mejoría en las primeras etapas de almacenamiento de los coques metalúrgicos correspondientes a la serie $\mathrm{Au}$ (Fig. 5).

\section{CONCLUSIONES}

La mayor o menor capacidad de un determinado carbón para interaccionar con el medio, provocando cambios químico-estructurales en la matriz orgánica que dan lugar a una alteración de las propiedades coquizantes en mayor o menor extensión, influye en su comportamiento durante la transformación del carbón a coque y, por consiguiente, en las propiedades del coque obtenido. Este hecho da lugar a que la alteración meteórica de los carbones provoque una pérdida de fluidez que no siempre está acompañada de un empeoramiento de los parámetros de calidad de los coques obtenidos.

Cuando una mezcla industrial experimenta pequeñas variaciones de sus propiedades y de la calidad de los coques resultantes como consecuencia de períodos de almacenamiento relativamente cortos, éstas se pueden explicar por los efectos contrarios y compensatorios que sufren determinados carbones que la componen, como se pone de manifiesto en el comportamiento de las dos series de carbones presentadas a modo de ejemplo. 

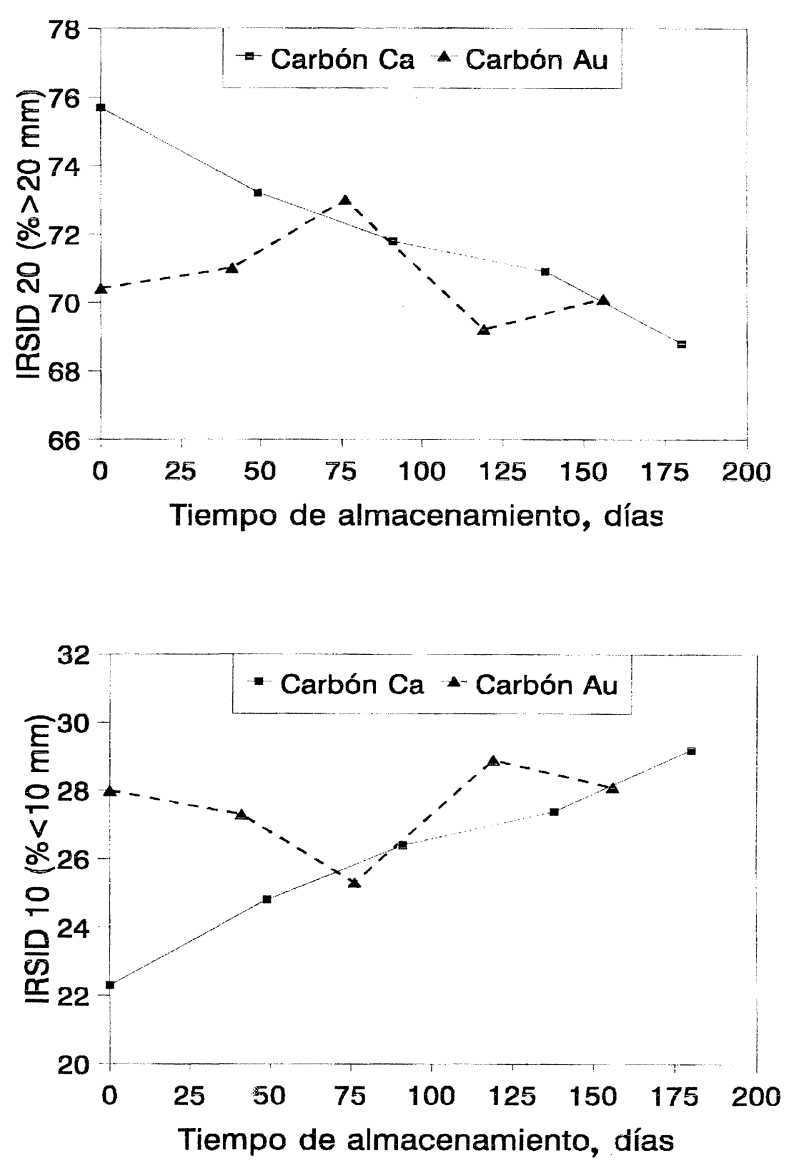

FIG. 4.- Variación de la resistencia mecánica (índices IRSID) con el tiempo de almacenamiento.

FIG. 4.- Variation of the mechanical strength (IRSID indices) with weathering time.

\section{Agradecimiento}

Los autores agradecen a la Comunidad Económica del Carbón y del Acero (CECA) la financiación de este trabajo (Proyecto 7220/EB-755), y a CSI Planos, S.A. (Ensidesa) el suministro de los carbones utilizados.

\section{REFERENCIAS}

(1) Nelson, C.R. (Ed.). Chemistry of Coal Weathering. Coal Science and Technology 14. Ed. Elsevier Science. Amsterdam, 1989.

(2) Davidson, R.M. Natural oxidation of coal. IEA Coal Research. Londres (R.U.), 1996.

(3) Ingram, G.R. y Runstadt, J.D. Fuel, 63, 1984: 292-296.

(4) Crelling, J.C., Schrader, R.H. y Benedict L.G. Fuel, 58, 1979: 542-545.

(5) Wu, M.M, Robbins, G.A., Winschel, R.A. y Burke F.P. Energy Fuels, 2, 1988: 150-157.

(6) Rouzaud, J.N., Jehlicka, J., Faugere, A.M., Pis J.J. y Alvarez R. Fuel, 73, 1994: 810-815.
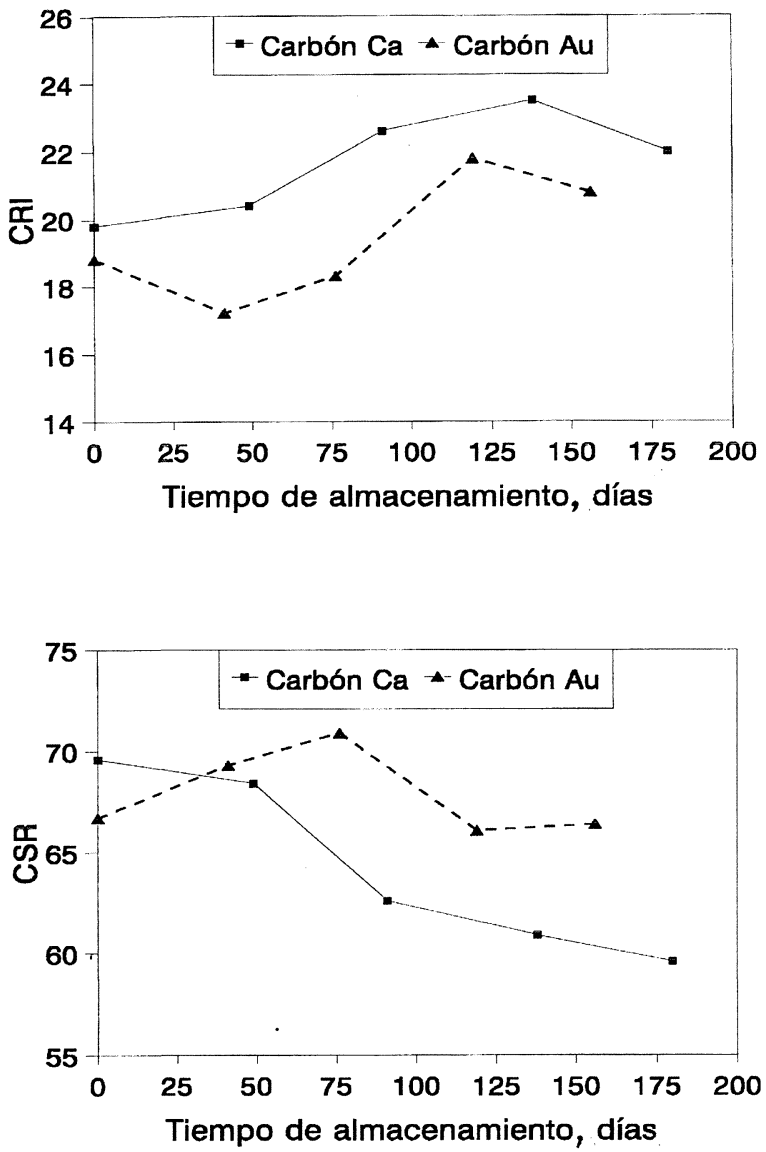

FIG. 5.- Variación de los índices CRI y CSR con el tiempo de almacenamiento.

FIG. 5.- Variation of CRI and CSR indices with weathering time.

(7) Huggins, F.E., Huffman, G.P., Dunmyre, G.R., Nardozzi, M.J. y Lin M.C., Fuel Processing Technol., 15, 1987: 233-244.

(8) Hiroyuki, S., Osamu, I. y Masashi, L. Fuel, 69, 1990: 317-321.

(9) Rhoads, O.A., Senfte, J.T., Coleman, M.M., Davis, A. y PAinTER, P.C. Fuel, 62, 1983: 1.387-1.392.

(10) Huffman, G.P., Huggins, F.E., Dunmyre, G.R., PignocCo, A.J. y Lin, M.C. Fuel, 64, 1985: 849-856.

(11) VAlia, H.S. ISS Ironmaking Conference Proceedings. Vol. 49. Chicago (EE.UU.), 1990: 199-209.

(12) Álvarez, R. Casal, M.D., Diez, M.A., Gonzalez, A.I., Lazaro, M., Suarez, C. y Pis J.J. Proc. Int. Conf. Coal Science. Ed. K.H. Michaelian. Vol. II. Banff (Canadá), 1993: 107-110.

(13) a) EsCUDERO, J.B. Bol. Inform. INCAR, 57. 1963: 51 págs. y b) Escudero, J.B. y Alvarez, R. Fuel, 60, 1981: 251-253.

(14) Painter, P., Starsinic, M. y Coleman, M. Fourier Transform Infrared Spectroscopy. Vol 4. Ed. Academic Press Inc. 1985: 169-241.

(15) Solomon, P.R., Hamblen, D.G. y Carangelo, R.M. Coal and Coal Products: Analytical Characterization Techniques. American Chemical Society Symposium Series 205. Ed. E.L. Fuller, Jr. Washington, D.C. (EE.UU.), 1982: 77-131.

(16) British Carbonization Research Association (BCRA). Report 87. Chesterfield (R.U.), 1980. 